\title{
Interplay Between Genomic Alterations and Androgen Receptor Signaling During Prostate Cancer Development and Progression
}

\author{
Michael D. Nyquist • Scott M. Dehm
}

Received: 16 November 2012 / Accepted: 2 January 2013 / Published online: 10 January 2013

(C) Springer Science+Business Media New York 2013

\begin{abstract}
Advanced prostate cancer $(\mathrm{PCa})$ treated with androgen deprivation therapy (ADT) eventually relapses to an ADT-resistant disease referred to as castrationresistant $\mathrm{PCa}$ (CRPC). Recent integrative analyses of $\mathrm{PCa}$ genomes have led to the elucidation of potential subtypes that are revelatory to the development of $\mathrm{PCa}$ as well as the mechanisms of resistance to ADT and CRPC progression. These studies have confirmed that alterations in the androgen receptor (AR) signaling axis are central to CRPC progression, and have uncovered complex mechanisms by which AR and other components of the AR signaling axis affect, and are affected by, genomic changes and epigenetic transformations. Among the most frequent alterations in CRPC are direct alterations in the AR gene. These AR gene alterations include AR amplification, point mutations, and more recently AR gene rearrangements leading to expression of truncated, constitutively active AR splice variants that are impervious to ADT. In this review, we will highlight genomic alterations that are important for development and progression of $\mathrm{PCa}$, with a focus on how these alterations affect, and are affected by, activity of the AR signaling axis.
\end{abstract}

\footnotetext{
M. D. Nyquist · S. M. Dehm $(\triangle)$

Masonic Cancer Center, University of Minnesota, Twin Cities, Mayo Mail Code 806, 420 Delaware Street SE,

Minneapolis, MN 55455, USA

e-mail: dehm@umn.edu

M. D. Nyquist

Graduate Program in Molecular, Cellular, Developmental Biology and Genetics, University of Minnesota, Twin Cities, Minneapolis, MN, USA

S. M. Dehm

Department of Laboratory Medicine and Pathology,

University of Minnesota, Twin Cities, Minneapolis, MN, USA
}

\section{Introduction}

Prostate cancer $(\mathrm{PCa})$ is the most frequently diagnosed male cancer and second leading cause of male cancer deaths in the USA [1]. The androgen receptor (AR) is a master regulator transcription factor in cells of prostatic lineage, and this master regulator function is maintained in $\mathrm{PCa}$ cells [2]. Therefore, the frontline treatment for locally advanced or metastatic $\mathrm{PCa}$ involves androgen deprivation therapy (ADT), which is achieved by blocking androgen production (surgical or chemical castration with LHRH agonists) or directly antagonizing the AR (antiandrogens). A major limitation is that castration-resistant or castration-recurrent prostate cancer (CRPC) results within 2-3 years due to alterations in the androgen/AR signaling axis. This limitation has begun to be addressed through mechanistic understanding of the changes to androgen/AR signaling that occur during disease progression. For example, it is now established that AR activity persists in CRPC despite ongoing $\mathrm{ADT}$, and the AR signaling axis remains a viable therapeutic target for treating CRPC patients.

New therapies that have been developed to re-target AR ligand binding and extend the lifespan of patients with CRPC include the cytochrome P450 c17 (CYP17) inhibitor abiraterone acetate [3], which inhibits androgen synthesis in testes, adrenals, and tumor tissue as well as the next generation antiandrogen enzalutamide/MDV3100 [4] which suppresses AR transcriptional activity even under conditions of AR overexpression. Despite these clinical successes, the development of resistance to abiraterone and enzalutamide, and ongoing disease progression for many patients, remains a major challenge. A greater appreciation of the complex genomic events involved in the initiation and progression of $\mathrm{PCa}$ is expected to yield insights into risk and likely routes of therapy resistance taken by individual cells in heterogeneous tumor populations. This could facilitate the individualization and 
optimization of treatment regimens, which are cornerstones in the concept of precision medicine. To this end, many studies investigating the PCa genome have identified disease- and/or progression-specific alterations in genes that impact the signaling and regulation of the androgen/AR signaling axis. Importantly, included in this list are a myriad of alterations in the AR gene at Xq11-12, which directly alter the regulatory dynamics and biological function of the AR.

\section{Recurrent Alterations in the PCa Genome}

Gene fusions between the AR-regulated TMPRSS2 gene promoter and the coding region of ETS- family transcription factors, including ERG and ETV1, are among the most frequent recurrent alterations in PCa. These fusions render ETS transcription factors androgen-responsive and under direct control of the AR signaling axis in PCa cells. Their existence in the earliest precursor lesions indicates that these are initiating drivers of $\mathrm{PCa}$ and efforts are being made to translate this knowledge into better diagnostic tools and new targeted therapies [5]. Knowledge of the broader genomic landscape of PCa has been greatly expanded in recent years by integrative whole-genome analyses using algorithms that coordinate datasets derived from studies of genome-wide copy number, exome sequence, mRNA expression, and epigenetic modification. These analyses have provided insights into the mechanisms by which mutations, epigenetic changes, and genome rearrangements conspire to dysregulate genes and pathways in $\mathrm{PCa}$. For example, a recent integrative study demonstrated that the $\mathrm{p} 53 / \mathrm{RB}$ pathway was disrupted in $34 \%$ of primary, hormone-naïve PCa and $74 \%$ of CRPC metastases, the PTEN/PI3K pathway was disrupted in $42 \%$ of primary $\mathrm{PCa}$ and $100 \%$ of CRPC metastases, and the RAS/RAF pathway was disrupted in $43 \%$ of primary PCa and $90 \%$ of CRPC metastases [6]. Similar integrative studies have found that MYC, WNT, FOXA1, SPOP, MLL2, CHD1, and NCOA2 are frequently affected by copy number alterations or point mutations [6-10]. Moreover, these integrative studies have revealed that AR copy number alterations and point mutations do not occur in primary PCa but occur in $58 \%$ of CRPC metastases [6]. However, when the broader AR signaling axis was considered, alterations were observed in $56 \%$ of primary PCa and $100 \%$ of CRPC metastases, confirming that this master regulator is among the most frequently altered pathways in PCa.

While these integrative studies have revealed important biological insights into $\mathrm{PCa}$, they have relied on targeted approaches that may not reveal the full spectrum of genomic alterations that could occur during disease development and progression. Indeed, recent whole-genome sequencing of seven primary $\mathrm{PCa}$ specimens and matched normal tissues demonstrated that important genomic events in $\mathrm{PCa}$ are likely underappreciated due to the limitations of targeted approaches such as $\mathrm{CGH}$ and exome sequencing. The most striking finding from $\mathrm{PCa}$ whole-genome sequencing was the high prevalence of copy number neutral, or balanced, translocations and inversions among the median 90 rearrangements per genome [9]. Some of these rearrangements had interrupted tumor suppressor genes such as PTEN and p53, which would be expected to activate oncogenic signaling through PI3K/Akt and RB pathways, respectively [9]. It is also interesting to note that some loci did not appear to undergo copy number loss even though inversions and/or point mutations were commonly observed, whereas other loci were frequently deleted [7, 9]. This suggests that specific chromatin configurations and/or genome architectures may be required for activation or inactivation of genes harbored within these affected regions.

Role of AR in the Genesis of Rearrangements in the $\mathrm{PCa}$ Genome

These integrative and whole-genome sequencing studies have raised an intriguing question: why is there a propensity towards structural alteration in the PCa genome? For TMPRSS2-ERG rearrangement-positive cancers, rearrangement breakpoints appear to be enriched near sites of AR and/or ERG binding with open chromatin histone marks, indicating that active transcription driven by these transcription factors may create chromatin environments that are prone to breakage [9]. There is also gathering evidence to support the notion that AR binding strongly influences higher-order chromatin structure and threedimensional organization of the nucleus in PCa cells, which may favor genesis of specific rearrangement events by enhancing proximity of discrete genomic domains [11-13].

The best evidence supporting a role for AR in shaping deterministic genomic events in the natural history of $\mathrm{PCa}$ progression comes from work investigating the mechanisms underlying recurrent TMPRSS2-ERG fusions. TMPRSS2 expression is controlled by an AR-driven enhancer element just upstream of the TMPRSS2 promoter [11]. This TMPRSS2 locus is $3 \mathrm{Mb}$ upstream of ETS family transcription factor ERG on chromosome 21. FISH-based studies with molecular probes targeting regions $5^{\prime}$ to the TMPRSS2 breakpoint and $3^{\prime}$ to the ERG breakpoint demonstrated that these loci are brought within close proximity in fusionnegative LNCaP PCa cells upon addition of the natural AR ligand, DHT [12]. DHT-induced proximity was also observed for the chromosomes 21 and 7 break fusion junctions of the TMPRSS2-ETV1 gene fusion [13]. Importantly, exposure of LNCaP or the normal prostate epithelial PrEC cell line to gamma irradiation, which induces genotoxic stress, synergized with DHT to induce genesis of 
TMPRSS2-ERG and TMPRSS2-ETV1 fusions [12, 13]. Induction of these fusion events appeared to require DHT/ARmediated recruitment of various DNA-directed enzymatic activities to the break fusion junctions, which promoted the formation of DNA double strand breaks at these sites. For example, activation-induced cytidine deaminase, the ORF2 endonuclease encoded by long interspersed nuclear elements (LINE-1), and topoisomerase IIB could all be recruited to these AR binding sites, and all have been shown to be important for generation of the fusion events [13, 14]. Importantly, DHT-induced proximity and irradiation-induced DNA damage were also shown to lead to generation of the SLC45A3: ETV1 fusion in LNCaP cells, which is a less-frequent PCa gene fusion leading to AR-mediated overexpression of ETV1 in a subset of $\mathrm{PCa}[13,15]$. These data imply that many other less frequently occurring rearrangements between androgenregulated genes and ETS family transcription factors may arise through this same mechanism [5]. Importantly, these ETS family fusions may then play a role in promoting further instability of the PCa genome. For example, ERG overexpression increases DNA damage in PCa lines and ERG binding is enriched at rearrangement breakpoints in primary $\mathrm{PCa}$, implicating ERG as a driver of subsequent genomic rearrangements and tumor progression $[9,16]$.

\section{$\mathrm{AR}$ and Epigenetic Alteration of the PCa Genome}

Epigenetic marks impart an additional layer of regulatory information on top of the information embedded in the $\mathrm{PCa}$ genome. Therefore, it is not surprising that many genomic studies have demonstrated that dysregulated epigenetic control accompanies the progression of PCa. Indeed, CRPC genomes display a general overall hypermethylation of CpG dinucleotides when compared with benign prostate [8]. Intriguingly, DNA methylation and genomic changes appear to coordinately alter the regulation of the cell cycle and testosterone metabolism, providing possible specific mechanisms through which DNA methylation promotes $\mathrm{PCa}$ progression [8]. Changes in the epigenetic regulatory environment can potentially change the physiology of the cancer cell and perhaps change the context in which existing and subsequent genomic events are manifest. One example of this is coordinate binding activity between AR and ERG. ERG represses AR transcriptional activity at loci involved in differentiation by recruiting the polycomb group (PcG) protein EZH2, which gives PCa cells more stem cell-like characteristics [17]. PcG proteins are responsible for maintaining bivalent repression domains to prevent the expression of lineage genes in embryonic stem cells [18]. Another recent study associated shared AR and ERG binding sites with transcriptional programs relating to movement and cell proliferation [19]. This study further showed that HDAC1, HDAC2, and HDAC3 and EZH2 bound to these AR/ERG binding sites [19]. This may have clinical relevance, as HDAC 1, HDAC 2, and HDAC 3 display high expression levels in a majority of $\mathrm{PCa}$ and $\mathrm{HDAC} 2$ levels in $\mathrm{PCa}$ tissues predict shorter PSA-free survival [20]. Mixed lineage leukemia 2 (MLL2), a H3K4-specific histone methyltransferase is mutated in $8.6 \%$ of PCas [7]. MLL2 is also commonly mutated in other cancers and is involved in the regulation of many signaling pathways including nuclear hormone signaling [21]. Indeed, MLL2 complex members could be immunoprecipitated with an AR antibody along with ERG and FOXA1 [7]. FOXA1 is mutated in $4 \%$ of PCas and is an important pioneer factor for the AR transcriptional program [22]. Interestingly, FOXA1 is preferentially recruited to binding sites enriched in $\mathrm{H} 3 \mathrm{~K} 4 \mathrm{me} 1 / 2$ marks, which in turn influences AR binding to nearby androgen response elements $[23,24]$. It is tempting to speculate that alterations in MLL2 activity could lead to global alterations in $\mathrm{H} 3 \mathrm{~K} 4 \mathrm{me} 1 / 2$ marks, which could in turn affect FOXA1 and AR binding and downstream regulatory processes in $\mathrm{PCa}$ cells.

\section{Truncated AR Splice Variants}

Overall, these genomic studies have demonstrated that the AR can be the instigator and the accomplice in effecting cascading genomic and epigenetic changes via chromatin interacting complex members. Interestingly, a pervasive role for the AR in driving deterministic events in $\mathrm{PCa}$ is further evidenced by the high rate of mutation and gene amplification of the AR gene in CRPC. These AR gene aberrations have been shown to alter AR signaling and regulation as well as facilitate AR transcriptional activity despite administration of targeted therapies designed to inhibit the AR [25-27]. For example, AR gene amplification leads to overexpression of AR protein, which sensitizes PCa cells to castrate levels of androgens, and can also elicit inappropriate agonist responses to antiandrogens such as bicalutamide [28]. Similarly, a myriad of point mutations have been described in the AR, many of which occur in the ligand binding domain (LBD) and broaden the repertoire of potential agonists [27, 29]. More recently, rearrangements that alter AR gene structure and splicing patterns have been described in PCa cell lines and xenografts [30, 31]. These AR splicing alterations underlie high expression levels of truncated AR variants that lack the AR LBD, which is the target of AR-centered therapies for PCa (Fig. 1). The role of AR splice variants in CRPC has been gaining importance in recent years due to the discovery of their prevalence in CRPC tissues [32-34], and their association with PCa progression and resistance to AR-targeted therapy [35-37].

Truncated AR splice variants were initially discovered in CRPC cell lines derived from the CWR22 xenograft model 


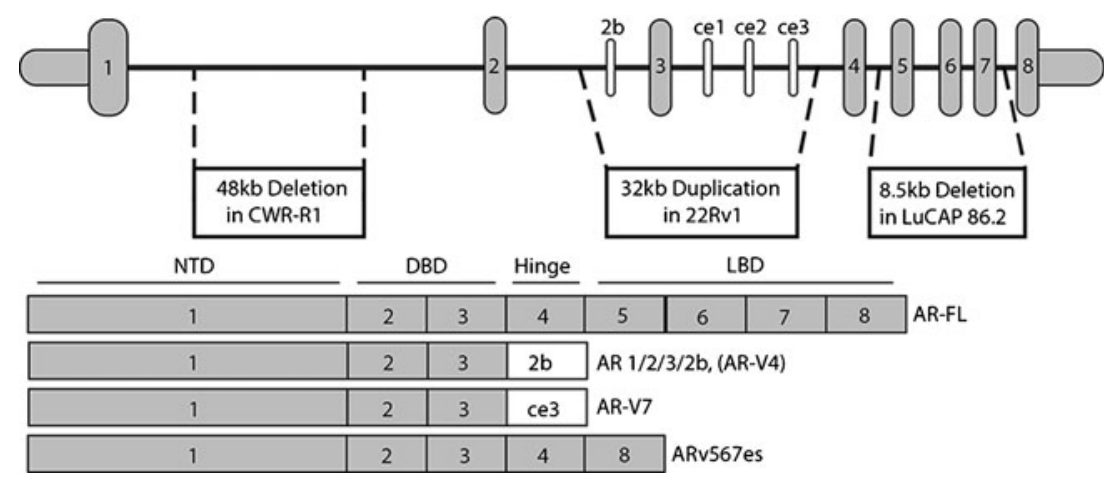

Fig. 1 A schematic of the AR gene structure is shown with cryptic exons (not to scale). Genomic regions involved in AR gene rearrangements associated with enhanced expression of truncated AR variants are indicated with dashed lines (above). The domain structure of full-

of PCa progression $[32,33,38,39]$. They are produced by aberrant splicing and premature translation termination, which results in synthesis of LBD-truncated, constitutively active forms of AR. To date, well over a dozen discrete AR splice variants have been described [40]. Identification and validation of protein translation of these $\mathrm{AR}$ variants in $\mathrm{PCa}$ cell lines has traditionally involved cloning the mRNA and designing AR variant sequence-specific siRNAs in order to achieve selective knockdown of that truncated AR variant $[32,39]$. This selective knockdown strategy has also facilitated functional characterization in PCa cell lines that naturally express high levels of truncated AR variants, such as the 22Rv1, CWR-R1, and VCaP cell lines. In these models, these strategies have revealed that truncated AR variants support androgen-independent expression of AR target genes and drive androgen-independent growth in a manner that is resistant to antiandrogens [32, 33, 35, 37, 41]. These key findings have highlighted the importance of understanding the role and origin of AR variants in clinical disease progression.

\section{AR Variant Splicing Mechanisms}

Recent investigation of the mechanisms underlying the synthesis of truncated AR splice variants in CRPC has highlighted genomic rearrangements as a previously unrecognized class of alterations affecting the AR gene [30, 31]. For example, our group reported that the $22 \mathrm{Rv} 1$ cell harbored a $32-\mathrm{kb}$ intragenic duplication of a segment encompassing AR exon 3, which harbors cryptic exons 2b, CE1, CE2, and CE3 (Fig. 1). All of these cryptic exons have been shown to splice with high efficiency downstream of AR exon 3 in this cell line; therefore, this rearrangement is an attractive explanation for these altered AR splicing patterns. Interestingly, the CWR22Pc cell line is an androgen-dependent subline derived from the same parental CWR22 xenograft as 22Rv1 cells. When CWR22Pc cells were cultured under castrate conditions, rare cells length $\mathrm{AR}$ and truncated $\mathrm{AR}$ variants with included exons are indicated (below). Regular AR exons are in gray, cryptic exons in white. $\mathrm{NH}_{2}-$ terminal domain $(N T D)$, DNA-binding domain $(D B D)$, ligand binding domain $(L B D)$

harboring this intragenic duplication emerged and continued to display enrichment over several weeks of culture in vitro. Importantly, outgrowth of these duplication-positive cells coincided with increasing expression of truncated AR variant mRNA and protein, indicating that expression of these species was restricted to the cell population harboring this underlying rearrangement in the AR gene [30]. These data provided the first clues that alterations in AR splicing may be due to underlying alterations in the structure of the AR gene.

An additional CRPC cell line in which truncated AR variants were identified and functionally characterized is the CWR-R1 cell line. In these cells, expression of the AR-V7 variant encoded by contiguously spliced AR exons $1,2,3$ and CE3 was shown to be enriched in CWR-R1 xenografts grown in castrated vs. intact mice [32]. We recently developed a strategy for paired-end massively parallel sequencing following hybridization-based enrichment of the AR locus, which revealed a 48-kb deletion in AR intron 1 in these cells [31]. Interestingly, quantitative analysis of this deletion indicated that the CWR-R1 cell line is heterogeneous, with a variable proportion of cells harboring this deletion. However, long-term culture under castrate conditions resulted in enrichment for this deletion-positive population to near-uniformity. As with the 22RV1 model, the expression level of the AR-V7 variant in the CWR-R1 cell line was directly proportional to the percentage of cells harboring this deletion [31]. Similarly, derivation of discrete subclones from this heterogeneous parental population revealed that high levels of truncated AR variant expression was restricted to the deletion-positive cell population [37].

In addition to these CRPC cell lines, there is also strong evidence to support a role for AR gene rearrangements in clinical CRPC metastases and xenograft tissues derived from clinical CRPC metastases. For example, deletions and duplications involving the AR gene directly alter gene copy number, and imbalances in copy number have been observed across the length of the AR gene in CRPC, but not 
primary prostatectomy specimens $[30,31]$. The $\mathrm{LuCaP} 86.2$ xenograft was derived following surgical resection of a CRPC bladder metastasis [41], and this tumor displayed reduction in copy number for AR exons 5-7 compared with flanking exons [31]. High-resolution analysis revealed deletion of an 8.5-kb segment encompassing AR exons 5-7 in approximately $50 \%$ of the cells within this tumor. Importantly, LuCaP 86.2 is the tumor model in which the truncated AR v567es variant was originally identified (es refers to exon skipping, whereby the splicing machinery skips AR exons 5-7, see Fig. 1) [41]. Although a clear cause-effect has not been demonstrated, it would be a logical to predict that the cells in this CRPC tumor harboring deletion of AR exons 5-7 are those cells expressing the AR v567es variant. The discovery of AR gene rearrangements may also explain why certain tumors favor expression of specific species of truncated AR splice variants. For example, it is tempting to hypothesize that ARv567es expression occurs in tumors with genomic alterations that impair proper splicing of exons 5-7, whereas AR-V7 overexpression arises through genomic rearrangements that promote more efficient splicing of AR exon CE3.

An alternative, non-mutually exclusive mechanism underlying synthesis of truncated AR splice variants could be acute, ADT-induced changes in regulation of AR splicing. Such a mechanism was revealed when blockade of fulllength $\mathrm{AR}$ expression or activity in late-passage $\mathrm{LNCaP}$ cells or VCaP cells resulted in acute increases in truncated AR-V7 protein levels [36, 42]. These acute splicing changes were rapidly reversed when expression or activity of fulllength AR was restored [42]. However, although AR-V7 levels were clearly increased under these conditions, total levels relative to full-length AR remained low and these cells remained dependent on full-length androgen signaling [42]. Nevertheless, given the importance of truncated AR variants in CRPC, it will be important to elucidate the mechanisms that regulate this plasticity in AR splicing and understand the interplay between these mechanisms and $\mathrm{AR}$ gene rearrangements in CRPC.

\section{Clinical Significance}

An important role for truncated AR variants in CRPC progression is supported by several studies demonstrating that metastatic CRPC displays high mRNA and protein expression of truncated AR variants compared with hormonenaïve prostatectomy specimens [32-34, 43]. Importantly, western blots of CRPC bone metastases demonstrated that a subgroup of CRPC tumors expressed nearly equivalent levels of full-length and truncated AR variants [34]. Moreover, these tissues displayed a higher AR immunostaining score, a higher proliferative index, and were associated with shorter survival after surgery ( 2 vs. 8 months) compared with CRPC tissues that expressed predominantly full-length AR [34]. This finding is in line with another study reporting that prostatectomy specimens displaying above-median ARV7 expression had a decreased probability of PSA progression-free survival [33].

The expression of truncated AR variants in a subset of CRPC may be especially relevant in light of new second line AR-targeted therapies that have recently been approved for patients with CRPC. Abiraterone and enzalutamide both increase overall survival of patients with CRPC, but resistance that exists de novo, or resistance that develops during therapy, is a major limitation for many patients. In mouse xenograft models, abiraterone slowed disease progression, but expression of the truncated AR v567es variant was associated with development of resistance [35]. Similarly, enzalutamide resistance is driven by truncated AR variants in the 22Rv1 and CWR-R1 cell lines, both of which harbor underlying AR gene rearrangements [37], and treatment of late-passage $\mathrm{LNCaP}$ cells or $\mathrm{VCaP}$ cells with enzalutamide leads to rapid increases in AR-V7 protein expression [36]. A complete understanding of the role of truncated AR variants in driving resistance to abiraterone and enzalutamide in patients will require a detailed and integrated analysis of AR gene structure and mRNA/protein expression patterns in tissues that display progression during treatment with these new agents.

\section{Biochemical Properties of Truncated AR Variants}

Nuclear localization of full-length AR is tightly regulated by ligand binding, and AR exists predominantly in the cytoplasm in the absence of androgens. Classical studies have demonstrated that AR nuclear import is supported by a bipartite nuclear localization signal located in the hinge domain encoded by AR exons 3 and 4 [44]. Therefore, truncated $\mathrm{AR}$ variants that do not harbor exon 4-derived sequence would be predicted to remain in the cytoplasm. However, loss of the AR LBD also eliminates the AR nuclear export signal. Recently, it has been shown that the net effect of losing both the canonical AR nuclear import and export signals results in constitutive nuclear localization of truncated AR variants in the absence of ligand [45]. In addition, this constitutive nuclear localization of truncated AR variants does not require full-length AR [45]. However, it has been demonstrated that the AR v567es variant can facilitate ligand-independent nuclear localization of fulllength AR through unclear mechanisms [41].

Ectopic expression of truncated AR variants in the androgen-dependent LNCaP cell line drives androgenindependent AR target expression [39, 41, 45] and growth under androgen-depleted conditions [32, 41, 42, 45]. Moreover, androgen-independent growth and AR target gene expression in AR splice variant-driven $22 \mathrm{Rv} 1$ and CWR- 
$\mathrm{R} 1$ cell lines are blocked when truncated AR variants are selectively knocked down. These data indicate that truncated AR variants drive androgen-independent growth by supporting ongoing AR transcriptional activity in CRPC cells. However, it has also been shown that truncated AR variants may have unique target gene specificities, perhaps due to the influence of variant-derived protein sequence located immediately at $\mathrm{COOH}$-terminal to the AR DBD. For example, unique targets have been ascribed to AR-V7, including AKT1 [32] as well as a set of M-phase genes including UBE2C, which is associated with CRPC progression [36]. However, some of these apparent differences may be due to differences in AR signaling output as opposed to true differences in target gene specificity. Indeed, UBE2C and other M-phase-associated target genes appear to be transcriptional targets of both full-length AR as well as truncated AR variants, but these genes display a biphasic pattern with induction at proliferative levels of AR signaling and repression at higher, anti-proliferative levels of AR signaling [37]. More recently, gene expression profiling experiments in CWR-R1 cells were designed to compare genes responsive to truncated AR variants or proliferative doses of DHT. In this set of experiments, the gene expression profile supported by truncated AR variants appeared to be a subset of the broader androgen/AR transcriptional program [37]. In future experiments, it will be important to elucidate the sets of genes that are regulated by truncated AR variants in clinical tissues, as this may reveal new therapeutic targets downstream of the AR signaling axis.

\section{Exploiting Genome Alterations to Define PCa Subtypes}

A major goal of cancer genomics is to understand the genomic changes that underlie the development and progression of $\mathrm{PCa}$. This is expected to improve prognostic and predictive accuracy for PCa patients. In particular, classification of clinically relevant subtypes is likely to prove invaluable in a clinical setting by informing treatment decisions. However, the exact criteria of what constitutes a subtype may be difficult to define. Separating tumors based on mutually exclusive genomic aberrations or a coordinated set of aberrations that accompany distinguishing physiologies and also have bearing on prognostic outcomes and treatment decisions would be ideal. However, even if tumors could be categorized into informative subgroups, $\mathrm{PCa}$ is a heterogeneous disease and it is possible that more than one subgroup may be present in a given individual. This is especially relevant in light of the observation that multifocal PCas often have different genomic rearrangements occurring between foci [46]. Additionally, metastatic PCa also appears to have clonal subgroups that respond differently to treatments [47]. Even more daunting is the possibility that mutually exclusive genetic events defined as subtypes in the development of PCa may not inform treatment decisions or have bearing on prognosis. Furthermore, genetic events that occur as an adaptive response to ADT may be the eminent factors to consider when choosing a therapy; this suggests it may be helpful to group cancers based on subtypes that result from acquired genomic changes causative of ADT resistance.

\section{PCa Developmental Subtypes}

TMPRSS2-ERG fusions occur in roughly half of all PCa. Therefore, one obvious PCa subtype is TMPRSS-ERG+/- or, more generally, the presence or absence of any AR-regulated or otherwise highly active gene promoter fused to any ETS family protein [5]. Recently, genomic studies have provided insights to mutations that appear to be important drivers in ETS- tumors. For example, SPOP mutations appear to be restricted to primary $\mathrm{PCa}$ and CRPC that do not harbor TMPRSS2-ERG fusions [7, 22]. Similarly, copy number loss or mutation of CHD1 was found to occur in $5.2 \%$ of tumors but most frequently in ETS- tumors [7]. Of note, CHD1 binds to $\mathrm{H} 3 \mathrm{~K} 4 \mathrm{me}$ histone marks and is commonly deleted in $\mathrm{PCa}$ contributing to an invasive cell phenotype [48]. ETS2, a putative tumor suppressor, is also deleted in tumors with the deletion of the $3 \mathrm{Mb}$ region between TMPRSS2 and ERG; however, this locus is also mutated at some frequency in TMPRSS2-ERG fusion-negative cancers [7].

SPINK1 overexpression is another PCa alteration that appears to be mutually exclusive with ETS+ cancers [49] and constitutes a subtype that occurs in $10 \%$ of all PCas. Notably, SPINK1 shares sequence homology with EGF suggesting that it could act as an EGFR agonist. Moreover, overexpression studies have demonstrated that SPINK1 mediates increased invasiveness in vitro [49], and knockdown of SPINK1 expression with shRNA or antibodies that neutralize SPINK1 have been shown to suppress cellular invasiveness [50]. Additionally, EGFR and SPINK1 suppression has an additive effect in inhibiting cellular invasion in SPINK+/ETS- cell line 22RV1 [50].

\section{PCa Treatment Subtypes}

Genomic changes that occur as a result of ADT may be useful for defining treatment-specific PCa subtypes. Because these would occur later in the natural history of $\mathrm{PCa}$ progression, these may be constrained or promoted by preceding genomic alterations that characterize specific $\mathrm{PCa}$ developmental subtypes (Fig. 2). For instance, it is unclear whether certain truncated AR variants would be able to interact and assemble into a functional complex with the same co-regulator proteins that interact with full-length AR. An example of this is FOXA1, which interacts with fulllength $\mathrm{AR}$ via the DNA-binding domain and hinge region 
Fig. 2 The relationship between PCa developmental subtypes is depicted on top. The relationship between developmental subtypes and treatment subtypes is largely unknown but subsequent changes to AR in response to ADT can be organized into the hierarchical categories depicted on the bottom

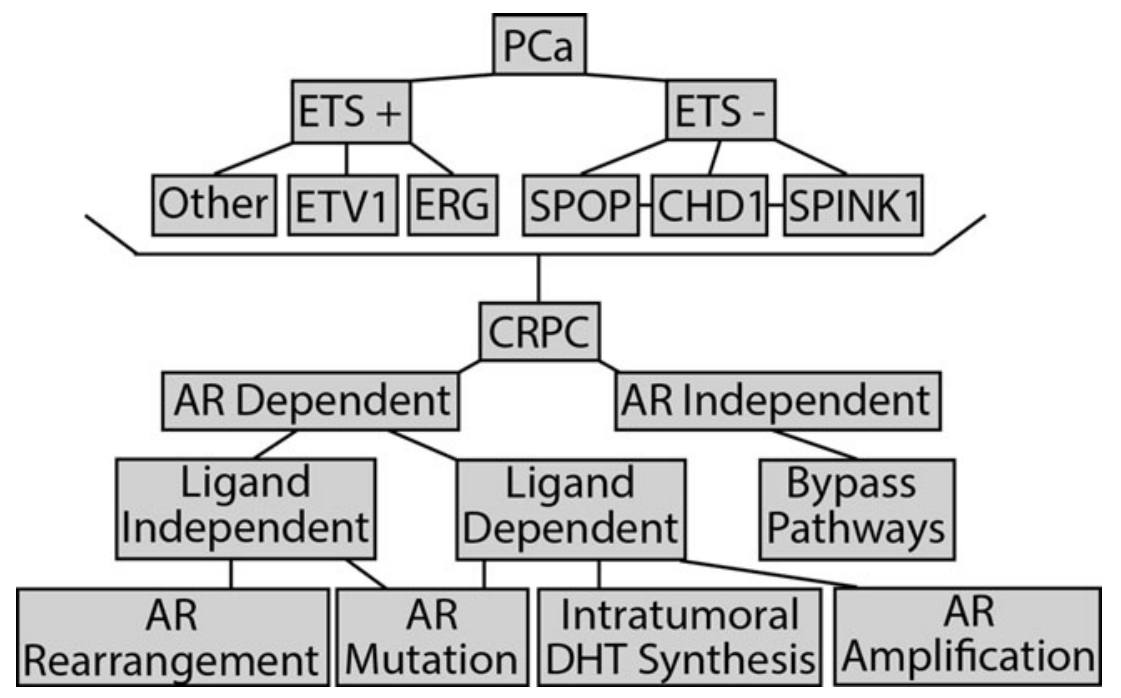

[51]. Because the AR hinge region is missing from most truncated AR variants (with the exception of ARv567es), it is possible that FOXA1 pioneer activity may not have the same impact on truncated AR variants as full-length AR. Similarly, the ETS factor ETV1 interacts with the AR ligand binding domain [52], which is absent in all truncated AR variants. Interestingly, many of the studies involving ectopic expression of truncated AR variants have been performed in the LNCaP cell line, which displays overexpression of ETV1, including a study which found that full-length AR was required for androgen-independent function of truncated AR variants [42]. In contrast, DAXX, which is involved in cellular differentiation and proliferation, regulates AR activity by binding to the AR NTD [53]. Therefore, DAXX may be able to interact with and regulate activity of truncated AR variants in a manner similar to full-length AR. Interestingly, SPOP has been shown to bind to and regulate DAXX [54], suggesting a potential interplay between SPOP and the AR in PCa. In addition, SPOP has also been shown to regulate SRC-3, an important AR co-regulator overexpressed in CRPC [55, 56].

Changes to the necessity or nature of AR signaling inductive to CPRC such as bypass pathways or changes to the AR itself such as mutations to change ligand specificity, amplifications to sensitize AR to ligand, and rearrangements that induce truncated AR splice variants may occur regardless of developmental subtypes and potentially be the dominant factor with regard to ADT response [25, 57]. For example, AR amplification can occur regardless of TMPRSS2-ERG fusion or other developmental subtype status [58]. However, it may be significant that AR gene amplification is associated with copy number gain of NCOA2 and a higher overall incidence of genomic copy number alterations [8]. AR gene amplification can also increase AR levels and sensitivity to residual DHT levels and lead to bicalutamide resistance [28]. Alternatively, inappropriately expressed biosynthetic enzymes that enable intratumoral synthesis of androgens, reviewed extensively elsewhere [59], are likely to drive ongoing AR signaling without structural changes to the AR gene locus. In these cases, it is anticipated that tumors harboring AR gene amplifications or altered expression of DHT biosynthetic enzymes would respond to therapy with abiraterone or enzalutamide. Indeed, enzalutamide was developed with the goal of addressing the challenge of treating CRPC driven by AR protein overexpression.

In contrast to amplification- or mutation-based alterations in full-length AR activity, evidence suggest that cancers driven by truncated AR variants may not require fulllength AR and would thus be resistant to treatments targeting the LBD or androgen synthesis [22, 36, 37]. This highlights the need to develop drugs that target the AR NTD or DBD, which are encoded by the first three exons of the AR gene. One such drug is EPI-001, which has been shown to bind the AR NTD and prevent transcriptional activity of full-length $\mathrm{AR}$ by blocking interactions with important $\mathrm{AR}$ coactivators [60]. Additionally, changes in growth factor signaling axes such as EGFR or IGF1, reviewed elsewhere [25], may activate AR signaling in the absence of ligand or bypass AR signaling altogether by activating mitogenic signaling pathways and render tumors resistant to antiandrogen therapies. Importantly, AR gene amplification events and AR gene rearrangement events may not be mutually exclusive, as AR gene copy number imbalances have been observed in CRPC xenografts and clinical metastases displaying overall increases in AR copy number [30, 31].

\section{Future Perspectives}

Recent advances in technology and increased sophistication have illuminated complex changes that occur in $\mathrm{PCa}$ and 
possible patterns that can be exploited therapeutically. However, AR alterations that drive ADT resistance, such as point mutations, copy number increases, and intragenic rearrangements have not been linked to other contextual alterations in the PCa genome. Whole-genome sequencing on CRPC metastases is anticipated to yield insights into the context and patterns of resistance to the expanding repertoire of drug options for ADT.

\section{References}

1. Siegel R, Naishadham D, Jemal A (2012) Cancer Statistics, 2012. CA Cancer J Clin 62(5):283-298

2. Garraway L, Sellers WR (2006) Lineage dependency and lineagesurvival oncogenes in human cancer. Nat Rev Cancer 6(8): 593-602

3. Bono JSD, Logothetis CJ, Molina A et al (2011) Abiraterone and increased survival in metastatic prostate cancer. N Engl J Med 364 (21):1995-2005

4. Scher HI, Fizazi K, Saad F et al (2012) Increased survival with enzalutamide in prostate cancer after chemotherapy. N Engl J Med 367(13):1187-1197

5. Rubin M, Maher C, Chinnaiyan AM (2011) Common gene rearrangements in prostate cancer. J Clin Oncol 29(27):3659-3668

6. Taylor BS, Schultz N, Hieronymus H et al (2010) Integrative genomic profiling of human prostate cancer. Cancer Cell 18 (1):11-22

7. Grasso CS, Wu Y-M, Robinson DR et al (2012) The mutational landscape of lethal castration-resistant prostate cancer. Nature 487 (7406):239-243

8. Friedlander TW, Roy R, Tomlins S et al (2012) Common structural and epigenetic changes in the genome of castration-resistant prostate cancer. Cancer Res 72(3):616-625

9. Berger MF, Lawrence MS, Demichelis F et al (2011) The genomic complexity of primary human prostate cancer. Nature 470 (7333):214-220

10. Beltran H, Yelensky R, Frampton GM et al (2012) Targeted nextgeneration sequencing of advanced prostate cancer identifies potential therapeutic targets and disease heterogeneity. Eur Urol S0302-2838(12):01006-01008

11. Wang Q, Li W, Liu XS, Carroll JS et al (2007) A hierarchical network of transcription factors governs androgen receptor-dependent prostate cancer growth. Mol Cell 27 (3):380-392

12. Mani R-S, Tomlins S, Callahan K et al (2009) Induced chromosomal proximity and gene fusions in prostate cancer. Science 326 (5957): 1230

13. Lin C, Yang L, Tanasa B et al (2009) Nuclear receptor-induced chromosomal proximity and DNA breaks underlie specific translocations in cancer. Cell 139(6):1069-1083

14. Haffner MC, Aryee MJ, Toubaji A et al (2010) Androgen-induced TOP2B-mediated double-strand breaks and prostate cancer gene rearrangements. Nat Genet 42(8):668-675

15. Tomlins S, Laxman B, Dhanasekaran SM et al (2007) Distinct classes of chromosomal rearrangements create oncogenic ETS gene fusions in prostate cancer. Nature 448(7153):595-599

16. Brenner JC, Chinnaiyan AM (2011) Disruptive events in the life of prostate cancer. Cancer Cell 19(3):301-303

17. Yu J, Yu J, Mani R-S et al (2010) An integrated network of androgen receptor, polycomb, and TMPRSS2-ERG gene fusions in prostate cancer progression. Cancer Cell 17(5):443-454
18. Richly H, Aloia L, Di Croce L (2011) Roles of the polycomb group proteins in stem cells and cancer. Cell Death Dis 2(9):e204

19. Chng KR, Chang CW, Tan SK et al (2012) A transcriptional repressor co-regulatory network governing androgen response in prostate cancers. EMBO J 31(12):2810-2823

20. Weichert W, Röske, Gekeler V et al (2008) Histone deacetylases 1, 2 and 3 are highly expressed in prostate cancer and HDAC2 expression is associated with shorter PSA relapse time after radical prostatectomy. Br J Cancer 98(3):604-610

21. Guo C, Chang CC, Wortham M et al (2012) Global identification of MLL2-targeted loci reveals MLL2's role in diverse signaling pathways. Proc Natl Acad Sci U S A 109(43):17603-17608

22. Barbieri CE, Baca SC, Lawrence MS et al (2012) Exome sequencing identifies recurrent SPOP, FOXA1 and MED12 mutations in prostate cancer. Nat Genet 44(6):685-689

23. Lupien M, Eeckhoute J, Meyer C et al (2008) FoxA1 translates epigenetic signatures into enhancer-driven lineage-specific transcription. Cell 132(6):958-970

24. Lupien M, Brown M (2009) Cistromics of hormone-dependent cancer. Endocr-Relat Cancer 16(2):381-389

25. Saraon P, Jarvi K, Diamandis EP (2011) Molecular alterations during progression of prostate cancer to androgen independence. Clin Chem 57(10):1366-1375

26. Steinkamp MP, O’Mahony O, Brogley M et al (2009) Treatmentdependent androgen receptor mutations in prostate cancer exploit multiple mechanisms to evade therapy. Cancer Res 69(10): 4434-4442

27. Gottlieb B, Beitel LK, Nadarajah A et al (2012) The androgen receptor gene mutations database: 2012 update. Hum Mutat 33 (5):887-894

28. Chen CD, Welsbie DS, Tran C et al (2004) Molecular determinants of resistance to antiandrogen therapy. Nat Med 10(1):33-39

29. Brooke GN, Bevan CL (2009) The role of androgen receptor mutations in prostate cancer progression. Curr Genom 10(1):18-25

30. Li Y, Alsagabi M, Fan D et al (2011) Intragenic rearrangement and altered RNA splicing of the androgen receptor in a cell-based model of prostate cancer progression. Cancer Res 71(6): $2108-2117$

31. Li Y, Hwang TH, Oseth L et al (2012) AR intragenic deletions linked to androgen receptor splice variant expression and activity in models of prostate cancer progression. Oncogene 31(45): 4759-4767

32. Guo Z, Yang X, Sun F et al (2009) A novel androgen receptor splice variant is up-regulated during prostate cancer progression and promotes androgen depletion-resistant growth. Cancer Res 69 (6):2305-2313

33. Hu R, Dunn T, Wei S et al (2009) Ligand-independent androgen receptor variants derived from splicing of cryptic exons signify hormone-refractory prostate cancer. Cancer Res 69(1):16-22

34. Hörnberg E, Ylitalo EB, Crnalic S et al (2011) Expression of androgen receptor splice variants in prostate cancer bone metastases is associated with castration-resistance and short survival. PLoS One 6(4):e19059

35. Mostaghel E, Marck BT, Plymate SR et al (2011) Resistance to CYP17A1 inhibition with abiraterone in castration-resistant prostate cancer: induction of steroidogenesis and androgen receptor splice variants. Clin Cancer Res 17(18):5913-5925

36. Hu R, Lu C, Mostaghel E et al (2012) Distinct transcriptional programs mediated by the ligand-dependent full-length androgen receptor and its splice variants in castration-resistant prostate cancer. Cancer Res 72(14):3457-3462

37. Li Y, Chan SC, Brand LJ, Hwang TH, et al. (2013) Truncated AR splice variants and enzalutamide resistance. Cancer Res. doi:10.1158/0008-5472.CAN-12-3630

38. Tepper CG, Boucher DL, Ryan PE et al (2002) Characterization of a novel androgen receptor mutation in a relapsed CWR22 prostate 
cancer xenograft and cell line characterization of a novel androgen receptor mutation in a relapsed CWR22. Cancer Res 62(22): 6606-6614

39. Dehm SM, Schmidt LJ, Heemers HV et al (2008) Splicing of a novel androgen receptor exon generates a constitutively active androgen receptor that mediates prostate cancer therapy resistance. Cancer Res 68(13):5469-5477

40. Dehm SM, Tindall DJ (2011) Alternatively spliced androgen receptor variants. Endocr-Relat Cancer 18(5):R183-R196

41. Sun S, Sprenger CCT, Vessella RL et al (2010) Castration resistance in human prostate cancer is conferred by a frequently occurring androgen receptor splice variant. J Clin Invest 120(8): 2715-2730

42. Watson P, Chen YF, Balbas MD et al (2010) Constitutively active androgen receptor splice variants expressed in castration-resistant prostate cancer require full-length androgen receptor. Proc Natl Acad Sci U S A 107(39):16759-16765

43. Zhang X, Morrissey C, Sun S et al (2011) Androgen receptor variants occur frequently in castration resistant prostate cancer metastases. PLoS One 6(11):e27970

44. Zhou ZX, Sar M, Simental JA et al (1994) A ligand-dependent bipartite nuclear targeting signal in the human androgen receptor. $\mathrm{J}$ Biol Chem 269(18):13115-13123

45. Chan SC, Li Y, Dehm SM (2012) Androgen receptor splice variants activate androgen receptor target genes and support aberrant prostate cancer cell growth independent of canonical androgen receptor nuclear localization signal. J Biol Chem 287(23): 19736-19749

46. Mehra R, Han B, Tomlins S et al (2007) Heterogeneity of TMPRSS2 gene rearrangements in multifocal prostate adenocarcinoma: molecular evidence for an independent group of diseases. Cancer Res 67(17):7991-7995

47. Ruiz C, Lenkiewicz E, Evers L et al (2011) Advancing a clinically relevant perspective of the clonal nature of cancer. Proc Natl Acad Sci U S A 108(29):12054-12059

48. Huang S, Gulzar ZG, Salari K et al (2012) Recurrent deletion of CHD1 in prostate cancer with relevance to cell invasiveness. Oncogene 31(37):4164-4170
49. Tomlins S, Rhodes D, Yu J et al (2008) The role of SPINK1 in ETS rearrangement-negative prostate cancers. Cancer Cell 13(6): $519-528$

50. Ateeq B, Tomlins SA, Laxman B et al (2011) Therapeutic targeting of SPINK1-positive prostate cancer. Sci Transl Med 3(72):72ra17

51. Gao N, Zhang J, Rao M et al (2003) The role of hepatocyte nuclear factor-3 alpha (Forkhead Box A1) and androgen receptor in transcriptional regulation of prostatic genes. Mol Endocrinol 17 (8):1484-1507

52. Shin S, Kim T-D, Jin F et al (2009) Induction of prostatic intraepithelial neoplasia and modulation of androgen receptor by ETS variant 1/ETS-related protein 81. Cancer Res 69(20):8102-8110

53. Lin D, Fang H, Ma A et al (2004) Negative modulation of androgen receptor transcriptional activity by Daxx. Mol Cell Biol 24 (24):10529-10541

54. Kwon JE, La M, Oh KH et al (2006) BTB domain-containing speckle-type POZ protein (SPOP) serves as an adaptor of Daxx for ubiquitination by Cul3-based ubiquitin ligase. J Biol Chem 281 (18):12664-12672

55. Li C, Ao J, Fu J et al (2011) Tumor-suppressor role for the SPOP ubiquitin ligase in signal-dependent proteolysis of the oncogenic co-activator SRC-3/AIB1. Oncogene 30(42):4350-4364

56. Wang Q, Carroll JS, Brown M (2005) Spatial and temporal recruitment of androgen receptor and its coactivators involves chromosomal looping and polymerase tracking. Mol Cell 19(5):631-642

57. Pienta KJ, Bradley D (2006) Mechanisms underlying the development of androgen-independent prostate cancer. Clin Cancer Res 12 (6):1665-1671

58. Bismar T, Yoshimoto M, Duan Q (2012) Interactions and relationships of PTEN, ERG, SPINK1 and AR in castration-resistant prostate cancer. Histopathology 60(4):645-652

59. Cai C, Balk SP (2011) Intratumoral androgen biosynthesis in prostate cancer pathogenesis and response to therapy. EndocrRelat Cancer 18(5):R175-R182

60. Andersen RJ, Mawji NR, Wang J et al (2010) Regression of castrate-recurrent prostate cancer by a small-molecule inhibitor of the amino-terminus domain of the androgen receptor. Cancer Cell 17(6):535-546 